He Astrophysical Journal, $203:$ L127-L129, 1976 February 1

1976. The American Astronomical Society. All rights reserved. Printed in U.S.A.

\title{
MASS LOSS IN GLOBULAR-CLUSTER RED GIANTS
}

\author{
Judith G. CoHEN \\ Kitt Peak National Observatory,* Tucson, AZ 85726 \\ Received 1975 September 2; revised 1975 October 22
}

\begin{abstract}
Echelle spectra of the brightest globular-cluster red giants, taken with the $4 \mathrm{~m}$ telescope at Kitt Peak National Observatory, reveal the presence of emission features at $\mathrm{H} \alpha$. These are interpreted as evidence for a circumstellar envelope, which is produced by mass loss. The gas is being lost at a velocity such that it is possible that the gas is escaping from the globular cluster. An estimate of the rate of mass loss $\left(2 \times 10^{-9} M_{\odot} \mathrm{yr}^{-1}\right)$ is obtained, which agrees well with that expected from revolutionary tracks for the difference in mass between red-giant stars and horizontal-branch stars in globular clusters.
\end{abstract}

Subject headings: clusters: globular - stars: circumstellar shells - stars: mass loss

\section{INTRODUCTION}

Strong theoretical evidence based on stellar evolutionary tracks predicts the existence of mass loss from stars in globular clusters between the red giant phase and the horizontal branch. Iben (1974) indicates that the best possible fit to the horizontal branch (HB) (and the RR Lyrae stars) occurs with a mass which is $0.1-$ $0.2 M_{\odot}$ less than the best estimate of the mass of stars on the red giant branch. Iben and Rood (1970) suggest that in the more metal-deficient clusters, the mean mass $(\Delta M)$ lost between the time the star begins to ascend the red giant branch and the time it reaches the $\mathrm{HB}$ is correlated with the metal deficiency of the cluster. For the most metal-deficient clusters such as M92, they expect $\Delta M$ to be quite small; for the more metal-rich clusters, $\Delta M$ will be larger.

A calculation is easily made of the amount of gas which can be accumulated through mass loss by all the red giants in a cluster over a period equal to the time between crossings of the galactic plane by the cluster $\left(t \approx 10^{8} \mathrm{yr}\right)$ and also over a period equal to the gasescape time $[t=$ radius of cluster/(velocity of gas escaping from the red giant) $\approx 10^{6} \mathrm{yr}$. With the parameters then available, Knapp, Rose, and Kerr (1973) expected to be able to detect this gas at $21 \mathrm{~cm}$, if it is neutral. However, they did not detect any gas. Furthermore, Hills and Klein (1973) did not see ionized hydrogen either, although their upper limits permit the accumulation of gas for times less than the interval between crossings of the galactic plane. These null results have provoked theoretical speculation that red giants may have coronae with a temperature of $10^{6} \mathrm{~K}$ (Hills 1973) or that something is seriously wrong with our conceptions of evolutionary tracks for globular cluster stars.

\section{OBSERVATIONS}

Using the echelle spectrograph on the $4 \mathrm{~m}$ telescope in 1975 May-June, eight spectra were obtained of four

* Operated by the Association of Universities for Research in Astronomy, Inc., under contract with the National Science Foundation. stars in three globular clusters. HD 122563 and HDE 232078, which are extremely metal-poor field red giants, were also observed. The spectra, obtained with a $50 \mu$ projected slit and a dispersion of $5.1 \AA \mathrm{mm}^{-1}$ at $5000 \AA$, were obtained with a cooled two-stage Carnegie image tube used with the Singer camera. The exposure time was 1 hour for the brightest of the globular cluster giants (B140 in M13 and M92 III-13). Calibration exposures using the spot sensitometer were made simultaneously with the stellar spectra and they were developed together. The spectra cover the wavelength range of 5000-7500 $\AA$.

A cursory examination of the globular cluster red giant spectra revealed that the $\mathrm{H} \alpha$ profiles were peculiar. Emission was seen, in some cases on the red wing of the line, in other cases on the blue wing, and in a few cases on both sides of the central absorption. There is no way that these emission features could be artifacts produced by the echelle spectrograph, the plate development, or the night-sky spectrum. Similar exposures of other stars have failed to show any emission. The spectra were traced using the PDS digital microphotometer and the calibration exposures to produce intensity plots. In cases where we had multiple exposures for a single star, the intensity scans were averaged to produce a final $\mathrm{H} \alpha$ profile. In Figure 1 we show the resultant profiles for the red giants B140 in M13, M92 III-13, M3 II-46, HDE 232078, HD 122563, and M13 IV-25. For the first star we have three spectra, and for the next three stars we have two spectra each. There is some indication from the spectra of B140 in M13 that the emission components in the $\mathrm{H} \alpha$ profile are time variable, but until more observations are accumulated this cannot be verified. The Na I D lines do not look peculiar.

For the two brighter field red giants, in HD 122563 we see no emission, whereas HDE 232078 shows strong emission. This is in conformity with the observations of Wallerstein et al. (1963), who also noted that the radial velocity of the hydrogen lines (based on six lines) in HDE 232078 is -10 ( \pm 4$) \mathrm{km} \mathrm{s}^{-1}$ with respect to the metallic lines. In M13 B140, this is also the case; we have found that the radial velocity of the central 


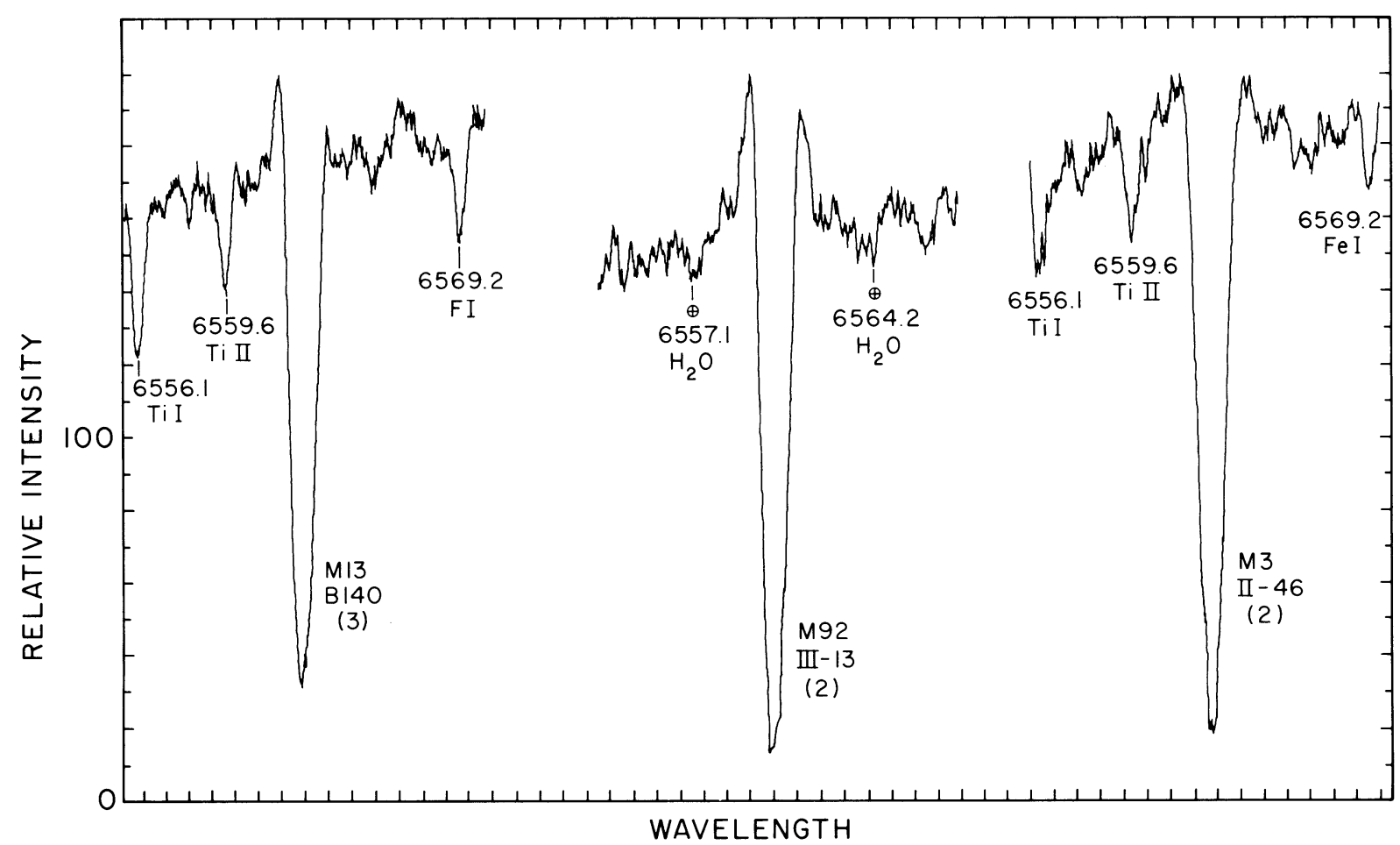

Fig. 1A

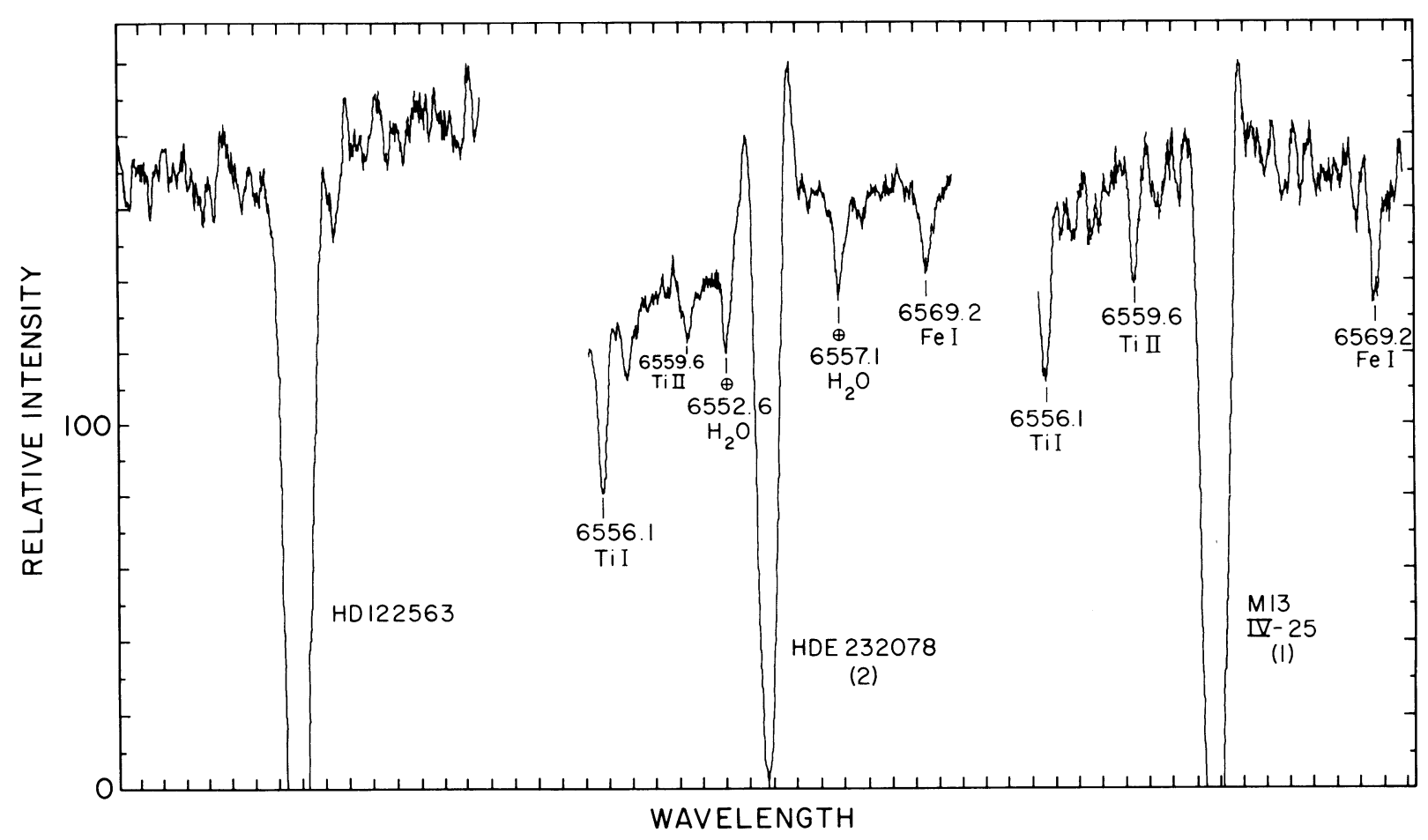

Fig. 1B

Fig. 1.-The $\mathrm{H} \alpha$ profiles of four globular-cluster red giants and two field red giants. Wavelength increases to the right, and the tick marks are $1 \AA$ apart. The intensity scale for each profile was multiplied by a constant so that the maximum intensity reached within the profile was the same for the six stars. The numbers in parentheses beneath the names of the stars indicate the number of spectra whose intensity tracings were added to produce the displayed profiles. The general slope of the continuum is due to the decline in intensity from the center of the echelle order coupled with the decline in sensitivity of the image tube toward the edge of the field. 
absorption in $\mathrm{H} \alpha$ is $-10( \pm 5) \mathrm{km} \mathrm{s}^{-1}$ with respect to the adjacent stellar lines in the same echelle order, while for M13 IV-25 and M3 II-46 no $\mathrm{H} \alpha$ velocity shift was seen. II It is not possible to do this with the red giants in more metal-deficient clusters as there are not enough stellar lines in any one order.

\section{DISCUSSION}

The $\mathrm{H} \alpha$ emission we have detected can arise only from a shell or circumstellar envelope. Since there is no possibility that this envelope could be formed from gas accreting onto the star, we conclude that the envelope must be formed from mass lost by the star. The apparent variation in $\mathrm{H} \alpha$ profiles with time for $\mathrm{B} 140$ in M13 is very suggestive of an expanding circumstellar envelope, as are the negative radial velocities for the $\mathrm{H} \alpha$ absorption in the two cases discussed above. Although it is true that emission is seen in some cases in the blue wing of the $\mathrm{H} \alpha$ line as well as the red (which is not the case for a classical P Cygni line profile), this may be due to the thin envelope and low rate of mass loss compared with P Cygni stars. The profiles we see are similar to those given by Sargent and Osmer (1969) for the F supergiant $89 \mathrm{Her}$, except that the gas is leaving that star at a much higher velocity. Deutsch (1969) has also detected mass loss from $M$ giants with an expansion velocity of $5-25 \mathrm{~km} \mathrm{~s}^{-1}$ (which is less than the escape velocity from near the photosphere), and this material is known in a few cases to extend to several hundred radii from the star, at which point its expansion velocity is greater than the local escape velocity.

The circumstellar envelopes must be very thin, as the photospheric line spectrum appears normal and the emission is not large. Also, Grasdalen (1975) reports that the infrared colors of the brightest globular cluster giants are normal. If we adopt the velocity of the emission wings of $\mathrm{H} \alpha$ as that typical of the expanding envelope, we obtain $V_{\text {exp }}$ approximately $45 \mathrm{~km} \mathrm{~s}^{-1}$. This is less than the escape velocity from the star near the photosphere, but presumably the remarks of the previous paragraph and Deutsch (1969) apply here also. We then conclude that since the escape velocity from globular clusters is $20-30 \mathrm{~km} \mathrm{~s}^{-1}$ (Hills and Klein 1973) the gas may actually leave the globular cluster. Furthermore, we can calculate a minimum value of the envelope mass, and hence of the mass loss. We assume that the envelope is optically thick in the Lyman lines and optically thin in the Balmer lines, that it is completely ionized, that it is composed only of hydrogen, and that the equation of continuity is satisfied. Then the density is given by

$$
n=n_{0}\left(R_{s} / r\right)^{2},
$$

where $r$ is the distance from the center of the shell assumed to start at $R_{s}=2$ stellar radii. Then the rate of mass loss is given by

$$
\frac{d M}{d t}=4 \pi M_{\mathrm{H}} V_{\exp } n_{0} R_{s}{ }^{2},
$$

where $M_{\mathrm{H}}$ is the mass of a hydrogen atom. The quantity $n_{0}$ may be obtained by noting that the emission seen at $\mathrm{H} \alpha, E_{32}$, is given by

$$
E_{32}=4 \pi h \nu n_{0}{ }^{2} \alpha(T) R_{s}{ }^{4} \int_{R_{s}}^{\infty} \frac{d r}{r^{2}} \operatorname{ergs~s}^{-1},
$$

where $\alpha(T)$ is taken as that for $10^{4} \mathrm{~K}$, and is not a strong function of the temperature (Pengelly 1964). Because $d M / d t$ is proportional to $R_{s}{ }^{0.5}$, the assumed value of $R_{s}$ does not have a large effect on the rate of mass loss. The emission we see has a minimum equivalent width of about $0.1 \AA$, which value includes only the emission visible on the spectra. A more likely estimate would be about $0.3 \AA$ to allow for the emission within the superposed emission and absorption profiles, but for illustrative purposes we shall use $0.1 \AA$ in the calculation. We use a stellar radius of $15 R_{\odot}$ (based on the luminosity and approximate effective temperature of the globular cluster giants). The result of this very crude approximation is a minimum mass-loss rate $d M / d t$ of $2 \times 10^{-9} M_{\odot} \mathrm{yr}^{-1}$. If this mass loss persists over a significant part of the red giant lifetime of $10^{8}$ years (Iben 1974), then the total mass loss will be 0.2 $M_{\odot}$. This is approximately the amount expected from evolutionary theory.

\section{CONCLUSIONS}

The $\mathrm{H} \alpha$ emission in globular-cluster red giants that we have seen is best explained by a circumstellar envelope produced by mass loss from the red giants. The $\mathrm{H} \alpha$ profiles may be variable with time and are variable from one red giant to another, probably even in the same cluster. From our limited sample, there is no apparent decrease in the strength of the emission for stars in more metal-deficient globular clusters. The expansion velocity is about $45 \mathrm{~km} \mathrm{~s}^{-1}$, so that a large fraction of the gas may leave the globular cluster, which may explain the difficulties radio astronomers are having in detecting the gas previously expected to be in globular clusters. The mass-loss rate, derived by very crude approximation, is approximately that expected from stellar evolutionary tracks between the red-giant branch and the horizontal branch.

We thank T. Gull for advice and instruction on the use of the echelle spectrograph.

\section{REFERENCES}

Deutsch, A. J. 1969, in Mass Loss from Stars, ed. M. Hack (Dordrecht: Reidel), p. 1.

Grasdalen, G. 1975 , private communication.

Hills, J. G. 1973, A p. Letters, 14, 69.

Hills, J. G., and Klein, M. J. 1973, Ap. Letters, 13, 65.

Iben, I., Jr. 1974, Ann. Rev. Astr. and Ap., 12, 215.

Iben, I., Jr., and Rood, R. T. 1970, Ap. J., 161, 587.
Knapp, G. R., Rose, W. K., and Kerr, F. J. 1973, A p. J., 186, 831. Pengelly, R. M. 1964, M.N.R.A.S., 127, 145.

Sargent, W. L. W., and Osmer, P. S. 1969, in Mass Loss from Stars, ed. M. Hack (Dordrecht: Reidel), p. 57.

Wallerstein, G., Greenstein, J. L., Parker, R., Helfer, H. L., and Aller, L. H. 1963, A p. J., 137, 280. 